Proceedings of the 2011 Winter Simulation Conference

S. Jain, R. R. Creasey, J. Himmelspach, K. P. White, and M. Fu, eds.

\title{
SIMULATING ENERGY EFFICIENT WIRELESS SENSOR NETWORKS USING CELLULAR AUTOMATA
}

\author{
Xiaoyun $\mathrm{Xu}$ \\ Xi Zhang \\ Long Wang \\ Department of Industrial Engineering and Management \\ College of Engineering \\ Peking University \\ Beijing, 100871, P.R.CHINA
}

\begin{abstract}
This paper studies a field coverage problem of wireless sensor networks. The objective is to prolong the network life for active sensing coverage. The problem is modeled using cellular automata. One deterministic algorithm and one probabilistic algorithm are proposed to extend the network life time. Both algorithms allow the activation of a particular sensor be determined by the current state of its immediate neighbors. Simulation examines both algorithms in percentage of coverage, residual energy and active sensors. The simulation results show a significant increase in network lifespan with a reasonable coverage through time. The results also indicate that a cellular automata is suitable for simulating large wireless sensor networks.
\end{abstract}

\section{INTRODUCTION}

Recent advances in affordable electronic components have a considerable impact on the development of wireless sensor networks, which constitute the platform of a large variety of applications including security, surveillance, health care and environmental monitoring. A wireless sensor network (WSN) is a special kind of network with distributed sensing and processing capability enabled by a group of devices called wireless sensors (Yick et al. 2008). When properly configured and deployed, these distributed sensors have the ability to monitor its surroundings and are capable of performing a limited set of processing activities such as numerical computing and wireless communication. A WSN tends to be autonomic and requires a high degree of cooperation between sensors to execute the tasks defined to the network.

Target detection is one of the major applications of WSNs. Detection is of interest for habitat monitoring, security, surveillance and other defense related tasks. The detection capability of the WSN is achieved through active monitoring the designated field. One of the fundamental metrics of interest in detection is the probability of missing a target, which can also be characterized by the percentage of the space being covered (Wang et al. 2007).

The monitoring task requires WSN to keep sensing its field of coverage, which continuously consumes energy. However, a critical aspect in WSN is power scarcity, driven mainly by battery power and limitations on weight (Cardei et al. 2005). The management of energy resources directly impacts the network operation lifetime and the performance of the application. Methods that optimize the sensor energy utilization therefore have great importance.

The main objective of this study is to identify the proper energy efficiency solution of the WSNs in the application of field monitoring. To achieve this, cellular automata are used to simulate algorithmic aspect of the sensors. Simulation has been developed to evaluate both deterministic and probabilistic algorithms according to their performance on energy consumption. The solution presented is based on the geographical position and the operational states of the sensors. 
$X u$, Zhang, and Wang

\section{LITERATURE REVIEW}

An important problem addressed in wireless sensor network literature is the sensor coverage problem. The coverage of a wireless sensor network indicates the sensors' ability to observe the physical space. The ideal coverage is achieved, according to (Cardei et al. 2005), through having each location in the physical space of interest within the sensing range of at least one active sensor.

Density control in WSNs is important to obtain the desired robust coverage. Density is typically measured by the percentage of active sensors in the WSN (Schurgers et al. 2002). While WSN with low density can suffer from insufficient coverage, WSN with too high density wastes excessive energy with little improvement in sensing coverage performance. Density control extends lifetime of the WSN by turning the redundant sensors to stand-by state and letting them wake up in the future to replace the energy-depleted or failing sensors. A properly designed density control algorithm is capable of prolonging network lifespan, while at the same time enhances system robustness in the presence of local sensor failure.

In energy efficient setting, active sensor selection algorithms are designed based on the specific problem requirements (e.g. percentage of coverage, connectivity, power efficiency). Different techniques have been proposed in literature (Carle and Simplot-Ryl 2004, Tian and Georganas 2002, Wang et al. 2003) for efficiently deciding the eligibility rule, that is, to determine if a sensor will be in stand-by state for the next round. The basic idea behind these algorithms is to design a mechanism such that each sensor can determine whether the target field has been collectively covered by other sensors. If so, the sensor will switch to the stand-by state for the current round.

A commonly adapted approach to achieve energy efficiency is through centralizing method, such as the ones proposed in Cardei and Du (2005) and Slijepcevic and Potkonjak (2001). Both works divide the sensors into disjoint sets, such that every set can individually perform the coverage tasks. These sets are then activated successively, and while the current sensor set is active, all other sensors are in the stand-by state. The activation command is broadcasted from a command center which can reach all the nodes simultaneously. The objective of these proposed approaches is to determine a maximum number of disjoint sets, as this has a direct impact on conserving sensor energy resources as well as on prolonging the network lifetime.

This study approaches the energy efficient field coverage problem from a distributed and localized view. Particularly, it is assumed that no central command center is in place to determine the behavior of each sensor in the WSN. The status of a sensor, instead, is solely determined by its current status and the status of its immediate neighbors. Cellular automata is introduced to simulate the behavior of the sensors with the objective of prolonging the active monitoring time span of the entire network.

The remainder of the paper is organized as follows. Section 3 presents the main concepts and formal definition of cellular automata. A detailed description of the field coverage problem as well as the simulation developed is presented in Section 4. The computational results are reported in Section 5. Finally, the concluding remarks and future works are presented in Section 6.

\section{CELLULAR AUTOMATA}

A cellular automaton (CA) is a discrete time decentralized system that can be used to model physical systems (Chopard and Droz 1998, Codd 1968, Goles and Martinez 1999). A unidimensional CA consists of a grid of cells with states that evolve in agreement with a set of rules. These rules are defined by the possible states of the adjacent cells.

CA is decentralized, discrete space-time systems that can be used to model physical systems. Cellular Automata are formally defined as quadruples $\langle d, Q, N, \delta\rangle$. The integer $d$ is the dimension of the space the cellular automaton works on. The dimension which is considered for this paper is two. $Q=\{0,1, \ldots, s-1\}$ is called the set of states. The neighborhood $N=\left(n_{1}, n_{2}, \ldots, n_{v}\right)$ is a $v$-tuple of distinct vectors of $Z^{d}$. The $n_{i}$ 's are the relative positions of the neighbor cells with respect to the cell, the new state of which is being computed. The states of these neighbors are used to compute the new state of the center cell. The local 


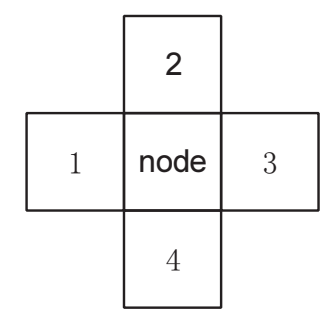

Figure 1: WSN neighborhood structure

function of the cellular automata $\delta: Q_{v} \rightarrow Q$ gives the local transition rule. A configuration is a function from $Z^{d}$ to $Q$. The set of all configurations is $Q^{Z^{d}}$. The global function $A$ of the cellular automaton on $C$ is defined via $\delta$ as follows:

$$
A(c, i)=\delta\left(c\left(i+n_{1}, \ldots, c\left(i+n_{v}\right)\right)\right), \forall c \in Q^{Z^{d}}, \forall i \in Z^{d}
$$

\section{MODELING FIELD COVERAGE PROBLEM}

This paper considers a field coverage problem in two dimensional space. The task of the WSN is to cover the entire field while at the same time reduce battery consumption. The basic structure of the simulation is a set of interconnected wireless sensors on a two dimensional lattice. Every sensor represents a cell of the cellular automata. The sensor is capable of monitoring the area of its own cell. The sensor has the following four attributes: 1) residual energy, 2) state (active or stand-by), 3) position (x and y coordinate) and 4) neighborhood structure. The residual energy is used to determine the amount of energy that remains in the sensor's battery. The state attribute determines whether the sensor is active or in the stand-by state. The position attribute is set at the beginning of the simulation and is fixed throughout the simulation. The sensors are deployed on a evenly distributed, bi-dimensional lattice and each sensor has exactly four immediate neighbors (see Figure 1). Sensors at the boundary(not in the corner) of the lattice only have three neighbors while sensors in the corner have two. To compensate this, one artificial neighbor is added for every boundary sensor and two are added for every corner sensor. These artificial neighbors are always in stand-by state and have zero residual battery power.

In simulation, the rules of the cellular automata are loaded from a separate file outside the simulator's code. This kind of implementation adds a greater flexibility to create new rules. The output of the simulation consists of a trace file containing 1) the state of the WSN obtained for a given experiment in terms of time elapsed, 2) global energy of the network (which is the summation of the residual energy of each sensor), 3) number of active sensors and 4) number of sensors alive. It is important to note that "number of sensors alive" also represents the effective coverage of the network, as only living sensors are capable of covering their own cell.

Each sensor is defined to have either of the two possible states: active or stand-by. In the active state, the sensor is monitoring an area equivalent to its cell. In the stand-by state, the sensor stop monitoring in order to save energy. The active state consumes significantly more energy than the stand-by state. The main objective is to design a set of proper algorithms to achieve a longer network lifetime while at the same time maintain a reasonably well monitoring capability. This objective, as will shown later in the algorithm design, is achieved by turning some of the sensors into stand-by state, such that fewer sensors are performing redundant monitoring task.

Two algorithms are examined in this study. One is a deterministic algorithm where the decision of each sensor is set by a deterministic function of the states of its four immediate neighbors. This is a classical case of the two dimensional cellular automata with totalistic rule (Schiff 2008). In particular, at each step, if a sensor observes that two or more of its neighbors are in the active state, it must change its state to the stand-by state to save energy. The sensor will remain stand-by for the current round, and in the next round, 
it will wake up and make another round of verification. If, however, it has less than two active neighbors at that moment, the sensor will set its state to active again. The pseudocode of the deterministic algorithm is shown in Algorithm 1.

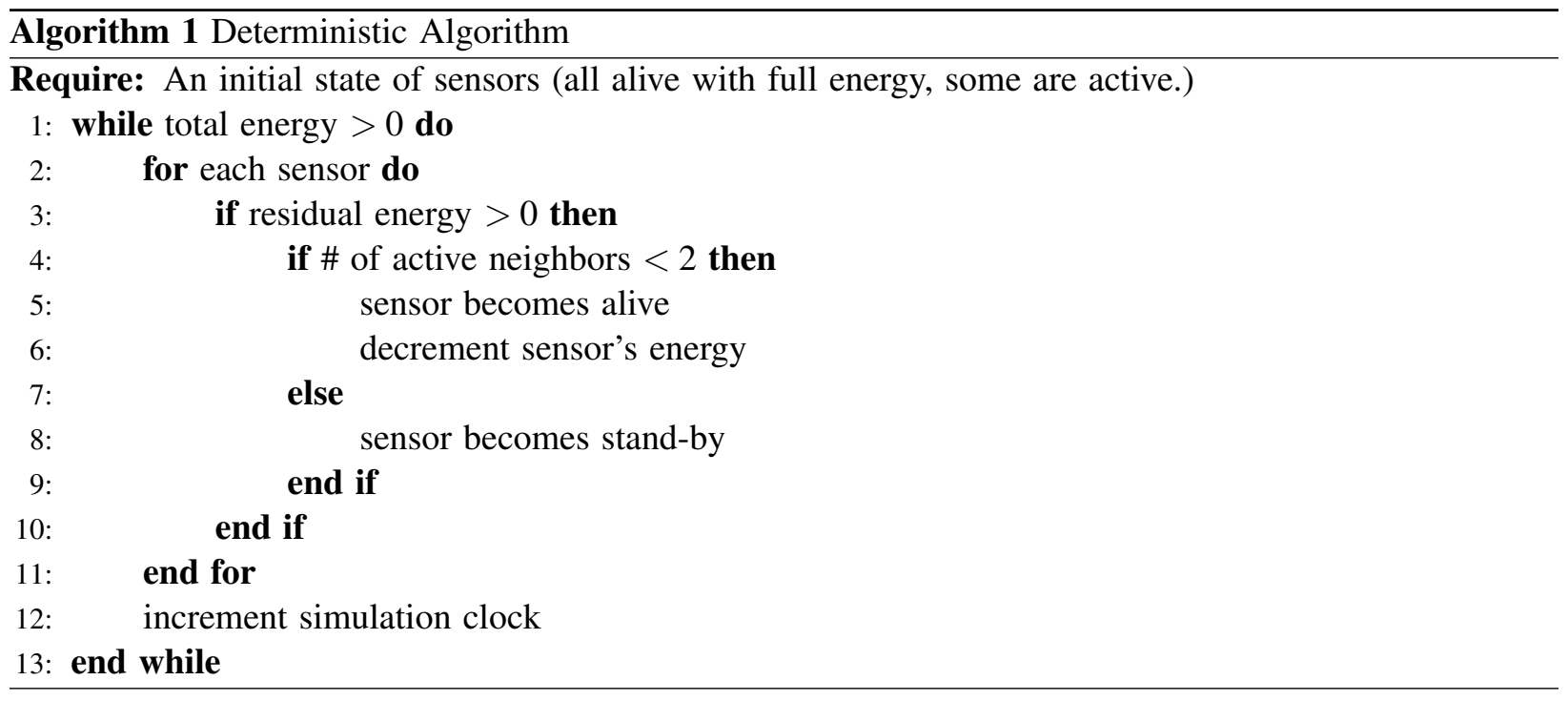

The other algorithm considered in this study is a probabilistic algorithm, which adds randomness to the sensors' decision. In this algorithm, the sensor's decision is affected by the states of its four immediate neighbors in a probabilistic fashion. When few of its neighbors are active, there is a higher probability that the sensor will decide to become active, whereas in the case when many of its neighbors are monitoring the field, the sensor is more likely to become stand-by state. Specifically, let the number of active neighbor at time $t$ be $n_{a}(t)$, then the decision probability is determined by the following function:

$$
\operatorname{Pr}(\text { sensor being active in } t+1)= \begin{cases}\frac{1}{n_{a}(t)} \times 100 \% & \text { if } n_{a}(t) \neq 0, \\ 100 \% & \text { otherwise. }\end{cases}
$$

The pseudocode of the probabilistic algorithm is presented in Algorithm 2.

Figure 2 shows a typical execution of the algorithm. At the first moment, all sensors in the grid are alive with their energy set to be full (Figure 2 (a)). The cell color from dark to white indicates the battery power goes from full to empty, respectively. At each step of the simulation, the sensors that are still alive make the neighborhood verification, and set their states according to the algorithm. Sensors consume a certain amount of energy when they are in active state. During the simulation, there will be an instant for each sensor when it will run out of energy. At that moment, the sensor is completely inactive and can not change its state anymore. As the simulation goes on, the number of active sensors will decrease (white cells in Figure 2(b)). Therefore, the coverage area of the WSN as well as the global energy will also decrease (Figure 2 (c)). The simulation terminates when all sensors have exhausted their energy (Figure 2(d)).

It is helpful to examine the possible behavior of the algorithm in the deterministic setting. In this simulation study, it is assumed that each sensor has two possible states (active or stand-by) and four immediate neighbors. For the deterministic algorithm there are total of $2^{4}=16$ rules for the sensors' behavior. Only 5 rules are assigned to turn on the sensor (set it to the active state), while the other 11 rules are used to turn it off (when it was verified that more than one adjacent cell contained an active sensor). This mechanism demonstrates the basic idea behind energy saving strategy.

\section{SIMULATION RESULTS}

In this study, the following three metrics are used to evaluate the two proposed algorithms: 


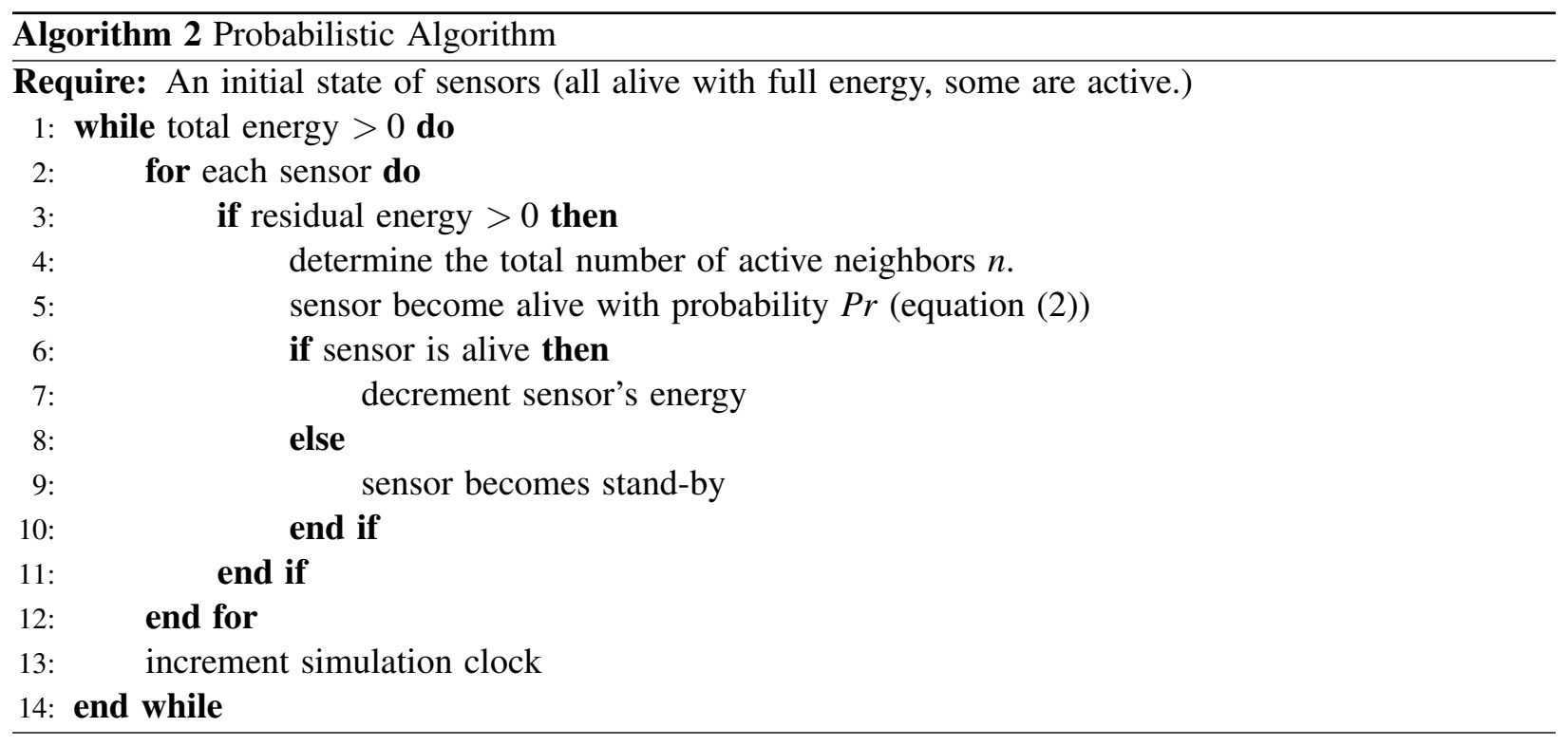

1. Energy of the WSN: the summation of the residual energy on all sensors over time;

2. Coverage Area: the percentage of the field monitored by the active sensors at each simulation step. Note that this is strictly proportional to the number of active sensors since each sensor can only monitor its own cell;

3. Network Lifespan: the time elapsed from the beginning of the simulation till all the sensors are dead (run out of energy).

The simulation is performed on a $50 \times 50$ lattice $(2,500$ wireless sensors $)$. Each sensor is assigned with an initial energy of 100 units and is set to either active or stand-by with equal probability in the beginning. Sensors in active state consume 1 unit of energy at each simulation step, whereas in stand-by they consume none. The sensor behavior is controlled by the deterministic and probabilistic algorithm proposed in the previous section. The entire simulation is coded using MATLAB and run on a laptop computer with $2.40 \mathrm{GHz} \mathrm{CPU}$ and $3 \mathrm{G}$ memory. The results presented in this section are the average of 100 independent executions of the simulation with random start.

It is not difficult to construct a naive worst case in terms of energy consumption. In the worst case, every sensor in the WSN is set to active state when simulation starts, and remains being active until its battery runs out. The network lifespan is therefore 100 time unit according to the current simulation setting.

Recall that the main objective of the proposed algorithms is to save energy while at the same time monitoring an area as large as possible. When sensors die, the capacity of monitoring the initial field decrease. The ideal situation is to monitor a largest possible area until all sensors have run exhausted their energy.

Figure 3 shows the network coverage result of the deterministic algorithm. During the most part of the simulation, the percentage of area covered oscillates between $70 \%$ and $30 \%$. This dramatic oscillation results from the sensing coverage setting. At an arbitrary simulation step $t$, a subset of sensors decide to switch their states accordingly to the algorithm. Since each sensor is only capable of monitoring its own cell, such switch will cause a dramatic topology change which leads directly to a coverage shift. It can be also be observed that the amplitude of oscillation decreases slightly as more sensors dies with time. The mean coverage stays around 50\% and plummets quickly as the life of the WSN approaches its end. The lifespan of the entire network is extended to 200 time unit with this deterministic algorithm, which two times longer than the naive worst case.

The coverage result of the probabilistic algorithm is shown in Figure 4. Unlike the deterministic one which is overly sensitive, the probabilistic algorithm exhibits a much more stable performance in terms of 


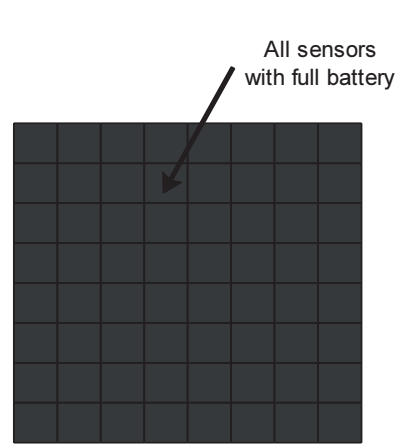

(a)

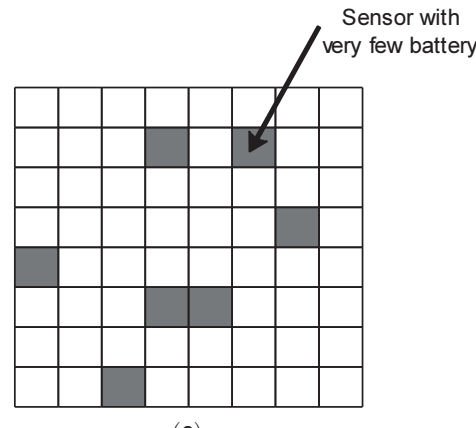

(c)

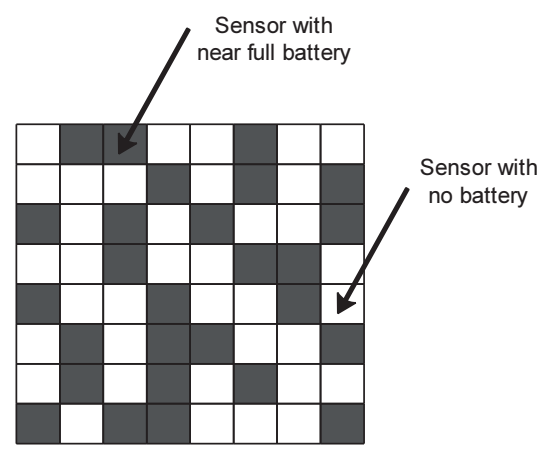

(b)

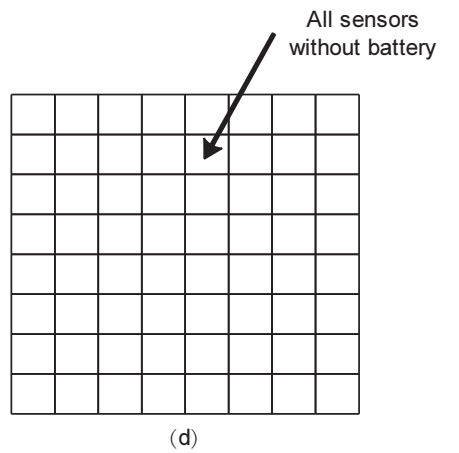

Figure 2: WSN simulation execution in cellular automata setting

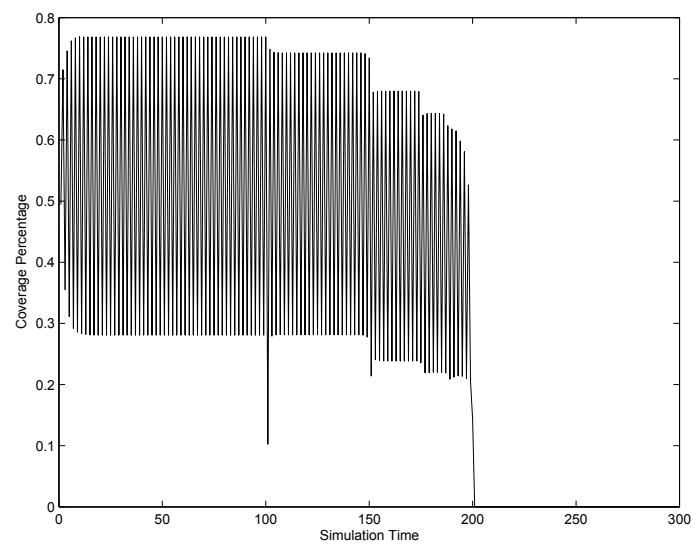

Figure 3: Field coverage with deterministic algorithm. 


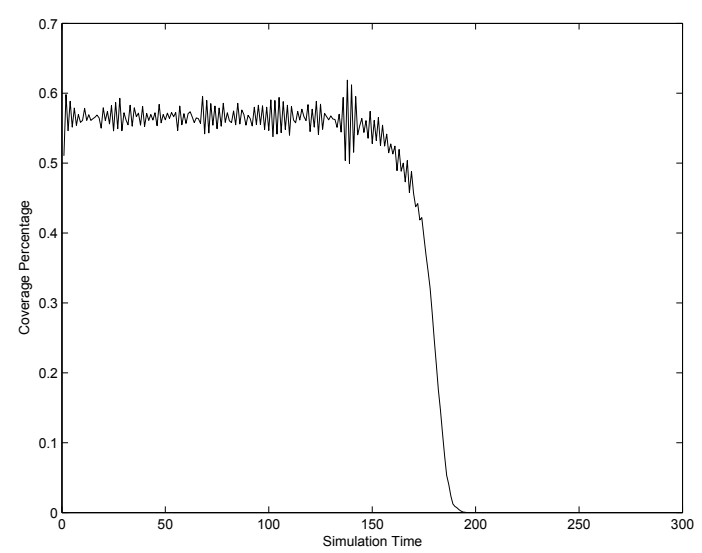

Figure 4: Field coverage with probabilistic algorithm.

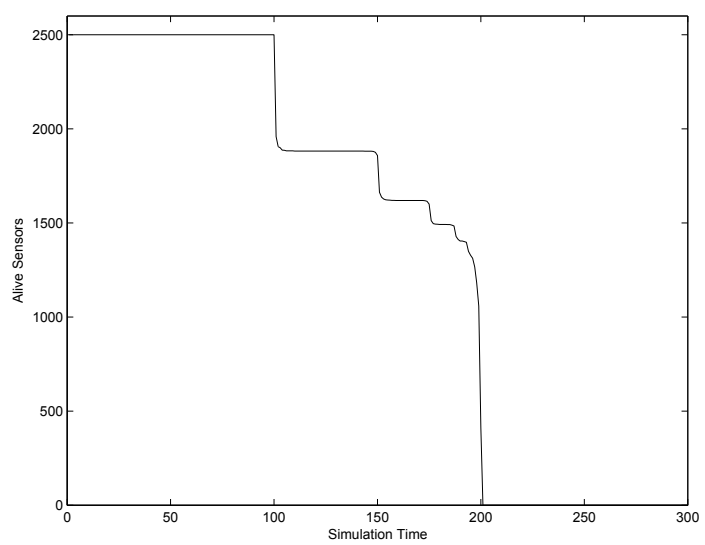

Figure 5: Number of living sensors with deterministic algorithm.

coverage. For most part of the simulation, the amplitude of oscillation is narrowed to $10 \%$. The average coverage performance maintains at the level of near $60 \%$ for approximately three quarters of the simulation and drops sharply near the end. The probabilistic algorithm results in a network lifespan of 200 time units, which is the same as the deterministic algorithm. The utilization of this algorithm increased by two times the network lifetime of the naive worst case.

Figures 5 and Figure 6 present the number of living sensors versus simulation time. In the deterministic case, it can be observed that number of the living sensors follows a stair-wise pattern against time. Significant drop of living sensors occurs at $1 / 2,3 / 4,7 / 8, \ldots$ of the network lifespan, while the number remains stable in between these points. In the probabilistic case, however, the line of living sensors is rather smooth. The number remains unchanged for approximately three quarters of the network lifespan. In both deterministic and probabilistic case, the dead sensors escalates in the last quarter of the lifespan, results in a significant drop in the field being monitored.

Figure 7 reports the amount of global energy of the network through simulation. Both algorithms show near linear energy depletion over time. However, the energy consumption of the probabilistic algorithm is slightly faster than that of the deterministic one. It is reasonable to concluded that in both cases the curves present similar behavior. This can be explained by the use of the linear declination function. At the tail of the energy consumption lines, it can be observed that when the network is left with very few living sensors, the probabilistic algorithm consumes less energy and tends to last longer, but this phenomenon is not universal and only appears near the end of the simulation. 


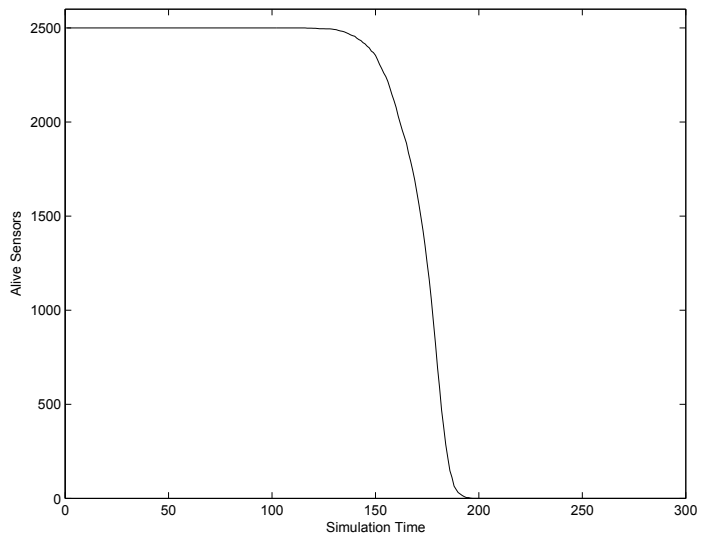

Figure 6: Number of living sensors with probabilistic algorithm.

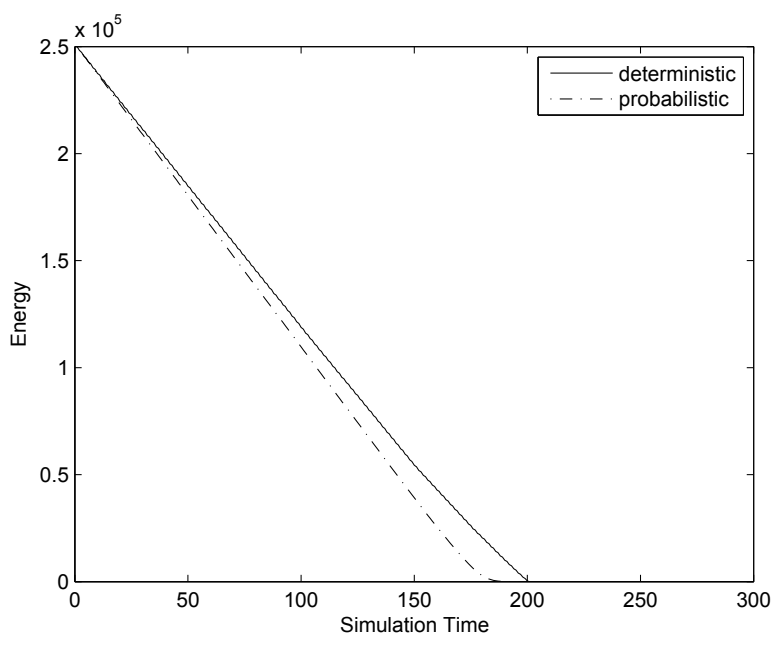

Figure 7: Energy with deterministic and probabilistic algorithm. 


\section{$X u$, Zhang, and Wang}

As expected, both algorithms show significant improvement in prolonging the network lifespan, while at the same time maintain the average coverage rate at around $60 \%$ to $70 \%$. The cellular automata simulation has demonstrated to be a viable alternative to develop and test WSN scenarios. Through cellular automata modeling, it is possible to test the impact of local decisions and observe global collective behaviors that might not be easy to identify through traditional analytical studies presented in the literature. As shown in Section 3, the construction of cellular automata simulation usually requires only the definition of the individual behavior of a cell and its corresponding neighborhood structure. Such structure typically requires much less effort to construct and is computationally very efficient and numerically stable due to its simplicity nature. Since the interconnection between cellular automata are much less restrictive, the simulation can accommodate very flexible designs. Numerous literatures such as Chopard and Droz (1998), Codd (1968), Goles and Martinez (1999) have demonstrated that simple local cellular designs can exhibit very complex global features that are usually difficult to obtain using the traditional discrete event method or Mont Carlo method.

\section{CONCLUSION}

This study presents a new approach to simulate large wireless sensor networks using cellular automata. This modeling approach allows flexible design of distributed and localized decision pattern, and is capable of creating scenarios with a huge number of sensors with reasonable computation overhead. Cellular automata can be used to indicate behavior tendencies and possible abnormal situations in large scale systems that could be difficult to visualize on an analytical study or hard to implement with the traditional simulation tools.

The main objective of this study is to identify the proper energy efficiency solution of the WSNs in the application of field monitoring. Two algorithms are purposed and explored. One is a deterministic algorithm who activates sensors when a fixed number of their immediate neighbors are not monitoring. The other is a probabilistic algorithm who operates in a similar way but with the activation probability strictly proportional to the number of its neighbors being stand-by or dead. The simulation results show that the deterministic algorithm produces rather intensive topology shifts and results in unstable monitoring, while the probabilistic algorithm shows a much more stable coverage performance. The average coverage percentage lies at approximately $60 \%$ to $70 \%$ through the most part of the network lifespan and plummets sharply as the network life approach its end. Both algorithms produce a network lifespan that is two times than the naive worst case where all sensors are set to be active through the entire simulation.

Currently, new features are being added in current simulation to 1) create more realistic scenarios and 2) enhance the performance of the current algorithm proposed. For example, it is observed from experiments that the jittering behavior of the deterministic algorithm can be alleviated through allowing the sensor to monitor all its neighboring cells instead of just its own cell. In addition, as suggested in Cunha et al. (2005), another metric that could be used to evaluate the algorithm would be the connectivity degree between sensors. This metric is important because a significant number of task are completed by letting all sensors in WSN communicate among themselves. More research should be conducted to evaluate the impact of the algorithms in the network connectivity degree.

\section{REFERENCES}

Cardei, M., and D. Du. 2005. "Improving Wireless Sensor Network Lifetime through Power Aware Organization". Wireless Networks 11 (3): 333-340.

Cardei, M., M. Thai, Y. Li, and W. Wu. 2005. "Energy-efficient Target Coverage in Wireless Sensor Networks". In INFOCOM 2005. 24th Annual Joint Conference of the IEEE Computer and Communications Societies, Volume 3, 1976-1984. IEEE.

Carle, J., and D. Simplot-Ryl. 2004. "Energy-efficient Area Monitoring for Sensor Networks". Computer 37 (2): $40-46$. 
Chopard, B., and M. Droz. 1998. Cellular Automata Modeling of Physical Systems. Cambridge University Press.

Codd, E. 1968. Cellular Automata. Academic Press, Inc. Orlando, FL, USA.

Cunha, R., A. Silva, A. Loreiro, and L. Ruiz. 2005. "Simulating Large Wireless Sensor Networks Using Cellular Automata". In Simulation Symposium, 2005. Proceedings. 38th Annual, 323-330. IEEE.

Goles, E., and S. Martinez. 1999. Cellular Automata and Complex Systems. Kluwer Academic Pub.

Schiff, J. 2008. Cellular Automata: A Discrete View of the World. Wiley-Interscience.

Schurgers, C., V. Tsiatsis, S. Ganeriwal, and M. Srivastava. 2002. "Optimizing Sensor Networks in the Energy-Latency-Density Design Space”. IEEE Transactions on Mobile Computing 1 (1): 70-80.

Slijepcevic, S., and M. Potkonjak. 2001. "Power Efficient Organization of Wireless Sensor Networks". In IEEE International Conference on Communications, Volume 2, 472-476. IEEE.

Tian, D., and N. Georganas. 2002. "A coverage-preserving node scheduling scheme for large wireless sensor networks". In Proceedings of the 1st ACM International Workshop on Wireless Sensor Networks and Applications, 32-41. ACM.

Wang, W., V. Srinivasan, K. Chua, and B. Wang. 2007. "Energy-efficient Coverage for Target Detection in Wireless Sensor Networks". In Proceedings of the 6th International Conference on Information Processing in Sensor Networks, 313-322. ACM.

Wang, X., G. Xing, Y. Zhang, C. Lu, R. Pless, and C. Gill. 2003. "Integrated Coverage and Connectivity Configuration in Wireless Sensor Networks". In Proceedings of the 1st International Conference on Embedded Networked Sensor Systems, 28-39. ACM.

Yick, J., B. Mukherjee, and D. Ghosal. 2008. "Wireless Sensor Network Survey”. Computer Networks 52 (12): 2292-2330.

\section{AUTHOR BIOGRAPHIES}

XIAOYUN XU is an assistant professor at Department of Industrial Engineering and Management, Peking University, P. R. China. He received his a Ph.D. in Industrial Engineering from Arizona State University, Tempe, Arizona. His research interests include optimization, algorithm design and discrete event simulation applied in manufacturing and service industries. His email address is xiaoyun.xu@pku.edu.cn.

XI ZHANG is an assistant professor at Department of Industrial Engineering and Management, Peking University, P. R. China. His research interests are in application of modeling and simulation in the area of quality and reliability. His email address is xi.zhang@pku.edu.cn.

LONG WANG is a professor at Department of Industrial Engineering and Management, Peking University, P. R. China. His research interests lies in control theory with the emphasis on the modeling and analysis of robots. His email address is longwang@pku.edu.cn. 